\title{
Modification of the BioMedicus centrifugal pump to provide continuous irrigation for neuroendoscopy: technical note
}

\author{
*Joyce Koueik, MD, Brandon G. Rocque, MD, MS, Jordan Henry, RN, BSN, Taryn Bragg, MD, \\ Jennifer Paul, RN, BSN, and Bermans J. Iskandar, MD \\ Department of Neurological Surgery, University of Wisconsin, Madison, Wisconsin
}

Continuous irrigation is an important adjunct for successful intraventricular endoscopy, particularly for complex cases. It allows better visualization by washing out blood and debris, improves navigation by expanding the ventricles, and assists with tissue dissection. A method of irrigation delivery using a centrifugal pump designed originally for cardiac surgery is presented.

The BioMedicus centrifugal pump has the desirable ability to deliver a continuous laminar flow of fluid that excludes air from the system. A series of modifications to the pump tubing was performed to adapt it to neuroendoscopy. Equipment testing determined flow and pressure responses at various settings and simulated clinical conditions. The pump was then studied clinically in 11 endoscopy cases and eventually used in 310 surgical cases.

Modifications of the pump tubing allowed for integration with different endoscopy systems. Constant flow rates were achieved with and without surgical instruments through the working ports. Optimal flow rates ranged between 30 and $100 \mathrm{ml} / \mathrm{min}$ depending on endoscope size. Intraoperative use was well tolerated with no permanent morbidity and showed consistent flow rates, minimal air accumulation, and seamless irrigation bag replacement during prolonged surgery. Although the pump is equipped with an internal safety mechanism to protect against pressure buildup when outflow obstructions occur, equipment testing revealed that flow cessation is not instantaneous enough to protect against sudden intracranial pressure elevation.

A commonly available cardiac pump system was modified to provide continuous irrigation for intraventricular endoscopy. The system alleviates the problems of inconsistent flow rates, air in the irrigation lines, and delays in changing irrigation bags, thereby optimizing patient safety and surgical efficiency. Safe use of the pump requires good ventricular outflow and, clearly, sound surgical judgment.

https://thejns.org/doi/abs/10.3171/2017.8.PEDS1726

KEY WORDS endoscopic irrigation; brain ventricles; ventricular endoscopy; surgical technique

I NTRAVENTRICULAR endoscopy has become an increasingly important tool in the armamentarium of the neurosurgeon. From CSF diversion to cyst drainage to tumor biopsy and resection, adult and pediatric neurosurgeons are using intraventricular endoscopy as a means to access deep-seated lesions with minimal damage to intervening brain tissue. ${ }^{2}$ Advances in endoscope technology have resulted in outstanding image quality and excellent illumination. However, to maintain excellent visualization, some form of irrigation is almost universally necessary.

Irrigation serves many purposes during intraventricular endoscopy. In complex endoscopic cases, irrigation helps prevent the accumulation of blood, air, and debris, thereby "maintaining a clear medium of image transmission and ventricular patency." 9 In both simple and complex endoscopic cases, irrigation can improve navigation by expanding the ventricles or preventing ventricular collapse, and it can assist with tissue dissection. ${ }^{8}$

Currently available methods for the delivery of irrigation have proven to be unsatisfactory for various reasons: the flow from an inflatable pressure bag slowly decreases as the irrigation bag deflates; there is an inevitable pause

ABBREVIATIONS ETV = endoscopic third ventriculostomy; ICP = intracranial pressure; rpm = rotations per minute.

SUBMITTED January 17, 2017. ACCEPTED August 8, 2017.

INCLUDE WHEN CITING Published online December 8, 2017; DOI: 10.3171/2017.8.PEDS1726.

* Drs. Koueik and Rocque contributed equally to this work. 
in irrigation when the bag needs to be changed; air is often trapped in the system and transmitted to the ventricles, which potentially compromises visualization; and it is not possible to assess flow rates and pressure buildup within the ventricle, which may negatively impact patient safety.

An alternative to the inflatable pressure bag is the peristaltic pump, which uses a roller or serial compression system to provide the motive force for the irrigant. A typical example is the intravenous infusion pump used routinely in hospitals. Infusion pumps are generally not capable of delivering the flow rates necessary for adequate irrigation of the ventricles in complex endoscopic surgery, and more sophisticated peristaltic pumps yield pulsatile flow patterns rather than the more desirable continuous flow and suffer from overpressurization of the circuit in the setting of a kink or obstruction. ${ }^{3}$

Here, we describe our modifications of a commonly used centrifugal perfusion pump to serve as the source of irrigation for intraventricular endoscopy. Introduced in 1978 for cardiopulmonary bypass, ${ }^{3}$ the BioMedicus pump provides continuous laminar flow that is driven by a magnetically coupled impeller-rotor head that prevents air from entering the distal pump tubing and provides a consistent and easily adjustable flow rate that is displayed on a digital readout. The impeller head (Fig. 1) has both inflow and outflow ports and is inserted into the interface with the pump itself. The flow rate is tightly controlled by regulating rotations per minute (rpm), and flow alarms are set at specific high- and low-flow rates; these features are particularly suitable for intracranial endoscopy.

\section{Methods}

We adapted the BioMedicus 550 Centrifugal pump (Medtronic) to serve as the source for intraventricular irrigation. Our modifications of the pump tubing include tubing and spikes for dual inflow from 2 irrigation bags, a Y-connector that allows both bags to feed into the impeller head, a flow meter built into the impeller outflow tubing, and a Luer lock mechanism that connects with the endoscope (Fig. 1). The assembly of all components in a sterile peel pack, which is custom made to our specifications, allows easy interface with commonly used endoscopes and simple setup for operating room staff.

The tubing system was custom packed to our specifications and is sold separately (University of Wisconsin Custom Pack \#5D56R5 Neuro Flows, Medtronic) with no financial interest by any of the authors.

Before using the pump system on patients, we performed a series of equipment tests in the laboratory to determine the performance of the pump and irrigation system under diverse, simulated surgical conditions. The goal of these experiments was to establish practical thresholds for the safe operation of the irrigation system in human ventricles. These experiments used the Olympus Infant Cystoscope with a 2.7- $\mathrm{mm}$ sheath with no separate irrigation, drainage, or instrument ports.

Subsequently, we used the pump in 11 consecutive, representative neuroendoscopic cases utilizing either the MINOP endoscope (Aesculap) with a 6-mm sheath or the Olympus Infant Cystoscope with a 2.7-mm sheath. We kept detailed prospective records of the flow rates, rpm, and all difficulties and complications related to irrigation. Notably, the MINOP endoscope has separate channels that house the endoscope, irrigation fluid, and instruments. The result is that, unlike the Olympus scope, introducing instruments into the MINOP scope would not be expected to alter the set irrigation flow rate. The data were analyzed retrospectively with institutional review board approval, and the results are discussed in a descriptive manner.

After the laboratory and clinical studies were completed, the pump was used routinely on ventricular endoscopic cases that required the rigid glass rod endoscopes described in this report. In our practice, the Olympus Infant Cystoscope is ordinarily used in simple endoscopic procedures, such as endoscopic third ventriculostomy (ETV) and septal fenestration, while the MINOP endoscope is used for more complex cases such as tumor resection and cyst fenestration. Cases that underwent shunt endoscopy utilizing a 1-mm flexible endoscope instead received syringe irrigation. Ringer's lactate solution $(130 \mathrm{mEq} / \mathrm{L}$ sodium, $4 \mathrm{mEq} / \mathrm{L}$ potassium, $3 \mathrm{mEq} / \mathrm{L}$ calcium, $109 \mathrm{mEq} / \mathrm{L}$ chloride, and $28 \mathrm{mEq} / \mathrm{L}$ lactate) was used as the irrigation fluid early in the series but was later replaced with normal saline solution $(0.9 \%$ sodium chloride injection, which contains $154 \mathrm{mEq} / \mathrm{L}$ sodium and $154 \mathrm{mEq} / \mathrm{L}$ chloride) for convenience. Artificial CSF is not used at our center. Following approval from the University of Wisconsin Institutional Review Board, data from a prospective database kept by the senior author (B.J.I.) and accompanying medical records, as needed, were retrospectively analyzed for any reports of major pump-related complications such as intracranial pressure (ICP) elevation, changes in vital signs, and failure of the pump system.

\section{Results}

\section{Equipment Testing in the Laboratory}

Several experiments were carried out under simulated surgical conditions to test the function of the pump and potential complications, especially with regard to flow consistency and effects on ICP.

First, we aimed to determine the consistency and fine control of the flow rate through the endoscope. Accordingly, we performed a series of tests on a BioMedicus pump connected to an Olympus Infant Cystoscope with a flow meter in series. From previous clinical experience, we have determined that the ideal flow rate for intracranial endoscopy is approximately $30-40 \mathrm{ml} / \mathrm{min}$ for the Olympus scope and $70-100 \mathrm{ml} / \mathrm{min}$ for the MINOP endoscope. We tested the irrigation flow rate through the Olympus scope, as determined by the flow meter at different pump speeds (rpm). The flow rate was consistent throughout the testing period. Gradual increases in pump rpm led to the fine control of flow through the scope, and changes in the flow rate as minuscule as $10 \mathrm{ml} / \mathrm{min}$ were easily achieved.

\section{Flow Into an Open System}

When continuous irrigation is used during neuroendoscopy, maintaining continuous outflow is necessary to prevent ICP elevation. Two main methods have been used to ensure that the irrigation fluid continuously flows out 


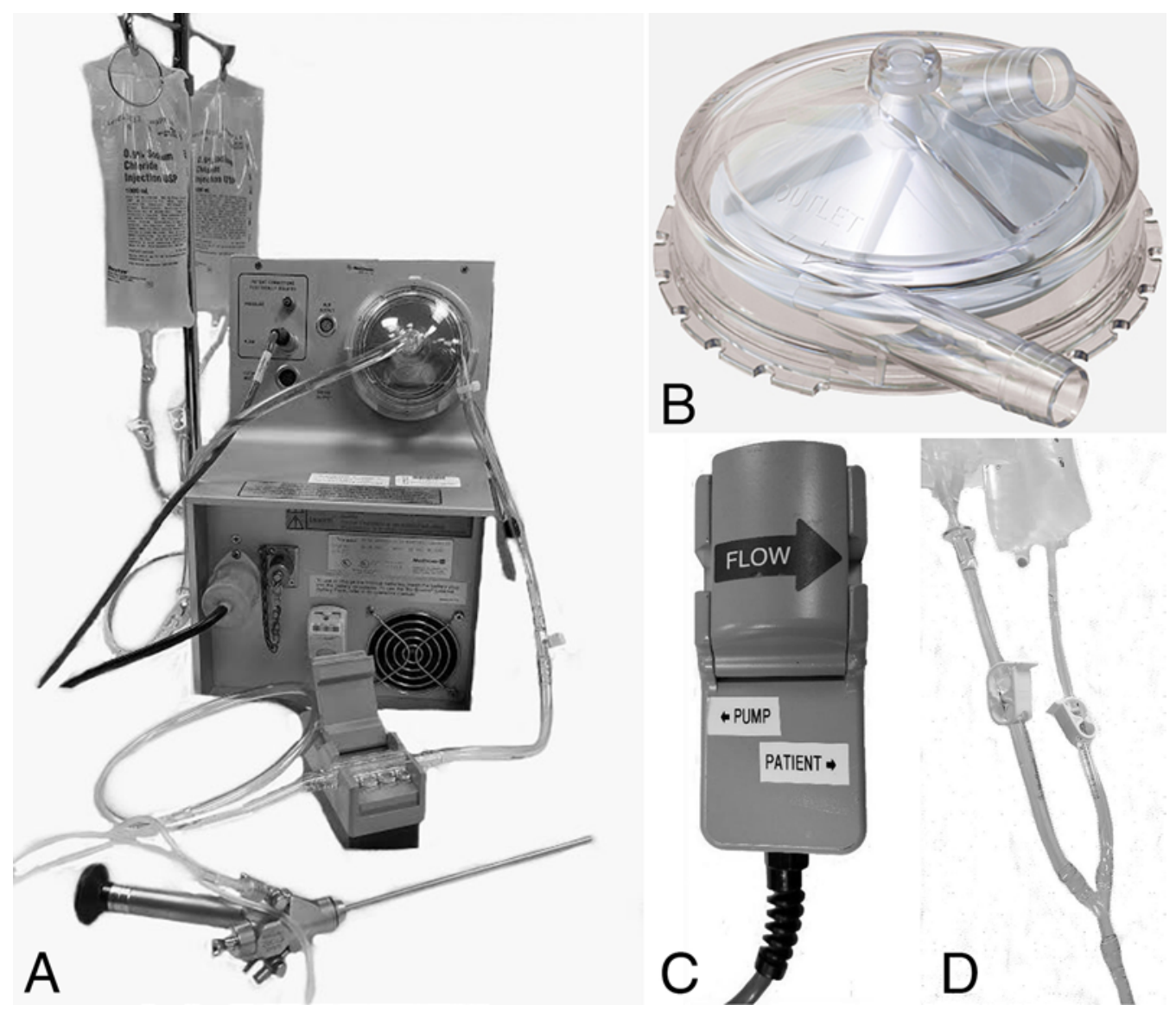

FIG. 1. BioMedicus 550 centrifugal pump. A: The entire connected pump assembly from the irrigation bags to the endoscope. $\mathrm{B}$ : The impeller head is inserted into the interface with the pump itself and creates differential pressure between the inflow and outflow ports, thereby resulting in continuous flow. C: The flow meter built into the impeller outflow tubing. D: Tubing and spikes that simultaneously draw from 2 irrigation bags connected to a Y-connector, thereby allowing both bags to feed into the impeller head. Figure is available in color online only.

of the cranial cavity: 1) some endoscopes have an outflow port; and 2) the endoscope is introduced into the ventricle through a peel-away sheath that allows the escape of fluid around the endoscope. Our next simulation aimed to examine the relationship between rpm and the flow rate into an open system reminiscent of a ventricle with outflow capacity, which consisted of a 1200-ml canister with a 19-Fr peel-away sheath acting as the outlet port (Fig. 2A). Throughout the simulation, the flow rate was consistent and directly proportional to rpm. Flow increased as rpm increased, a gradual increase in pump rpm led to the fine control of the flow through the endoscope (Fig. 2B), and changes in the flow rate as minuscule as $10 \mathrm{ml} / \mathrm{min}$ were easily achieved. This indicated that when consistent outflow is maintained and the irrigant is not allowed to accumulate in the canister (i.e., the ventricle), flow is consistent and can be finely controlled by the impeller speed.

\section{ICP in an Open System}

In clinical situations, flow is titrated as needed. While uncomplicated endoscopic surgery such as routine ETV requires little, if any, irrigation, bleeding requires irrigation to prevent clot formation in the ventricle. Therefore, our next step was to determine whether altering flow rates into an open system alters ICP. The sheath provides outflow around the endoscope shaft. In addition, the canister was fitted with a Codman ICP-monitoring device (Codman \& Shurtleff, Inc.). Pressure measurements were recorded as the pump flow was incrementally increased. Increasing rpm from 900 to 3000 resulted in a slight increase in pressure within the canister, but levels remained within a safe range (less than $20 \mathrm{~mm} \mathrm{Hg}$ ) (Fig. 2C). We concluded that in an open system unsafe elevations in ICP are unlikely to occur, even at high flow rates. Regardless, surgical judgment should be exercised, as high flow could cause direct damage to neighboring brain tissue.

\section{Flow Into a Closed System}

The BioMedicus pump is designed with a safety mechanism that interrupts flow if irrigation is pumped into a closed compartment. To evaluate the effectiveness of this mechanism in neuroendoscopy, the endoscope was connected to a balloon in a $1200-\mathrm{ml}$ closed canister with no outflow path (Fig. 3A). At an initial rpm of 800 and a flow of $100 \mathrm{ml} / \mathrm{min}$, the balloon started to expand. Over a period of 10 minutes, flow was noted to decrease as the balloon expanded (Fig. 3B). To maintain flow against balloon resistance, rpm was gradually increased to $1000 \mathrm{ml} / \mathrm{min}$. 


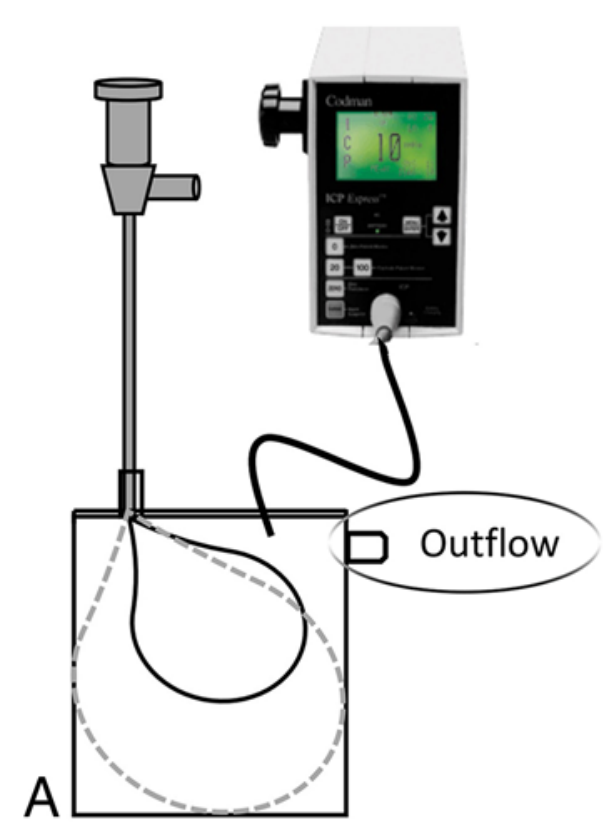

C
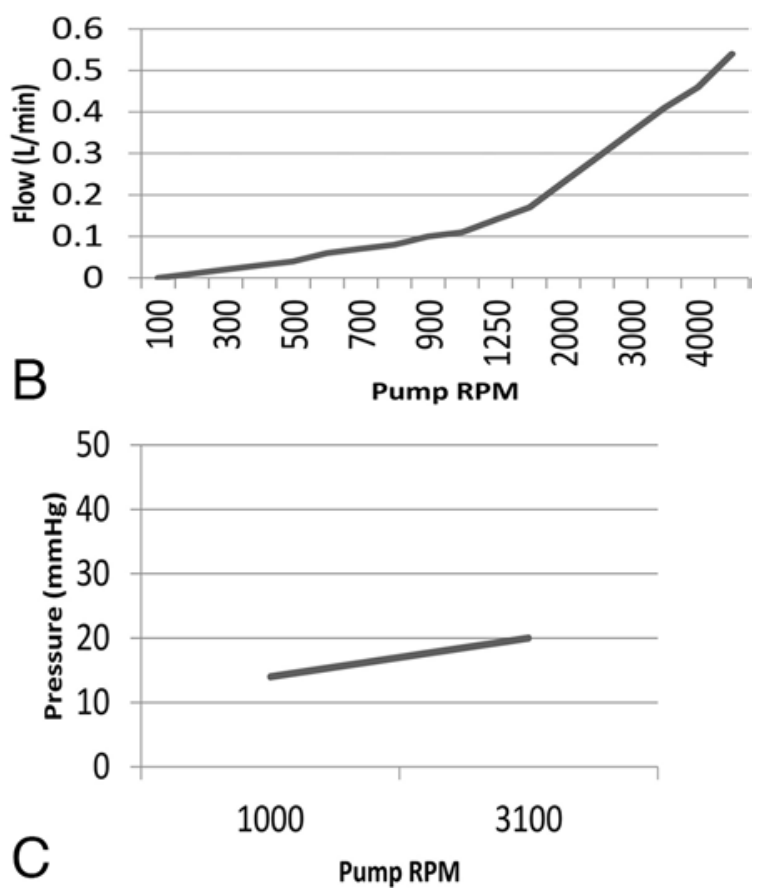

FIG. 2. Flow rate and canister pressure in an open system. A: Open system. An endoscope is connected to a 1200-ml canister with an open outflow track. A Codman ICP monitor is attached. B: Flow rate versus rpm. Flow increases as rpm increases. Gradually increasing pump rpm leads to fine control of the flow through the endoscope, and changes in flow rate as minuscule as $10 \mathrm{ml} / \mathrm{min}$ are easily achieved. C: ICP. In an open system, unsafe elevations in ICP are unlikely to occur even at high flow rates. This is equivalent to ICP with endoscopic irrigation flowing into the ventricles with an external drainage port. Figure is available in color online only.

Flow into the balloon continued for another 13 minutes before it was stopped when the balloon reached maximal expansion (i.e., the balloon touched the inner walls of the canister). We concluded that in a closed system with no outflow, inflow against resistance gradually decreases to a halt, which would theoretically prevent a rise in ICP in a clinical situation in which outflow is accidentally interrupted. However, this protective mechanism is not instantaneous and takes seconds to minutes before the flow actually stops in the face of outflow obstruction, which might cause ICP to rise.

\section{ICP in a Closed System}

Accordingly, to determine whether pumping fluid into a closed compartment causes a rise in ICP before the flow reversal safety mechanism kicks in, the pump system was connected to a closed canister and a balloon with no outflow, as described above, and the canister was fitted with a Codman ICP-monitoring device. Continuous pressure measurements were recorded with the pump running into a closed container (Fig. 3B). An initial pump speed of 900 $\mathrm{rpm}$ was selected to deliver a flow rate of $70-100 \mathrm{ml} / \mathrm{min}$. The reciprocal effect of increasing container pressure on the pump flow rate was then examined. With the pump running at $900 \mathrm{rpm}$ within the closed canister, ICP rose steadily. At 15 minutes, ICP rose to $20 \mathrm{~mm} \mathrm{Hg}$. Once ICP reached $30 \mathrm{~mm} \mathrm{Hg}$, the flow started to decline and eventually stopped after 8 minutes, but with an accompanying and gradual increase in ICP to $50 \mathrm{~mm} \mathrm{Hg}$. Subsequently, rpm was increased from 900 to 1500 to force fluid into the system at $100 \mathrm{ml} / \mathrm{min}$, which led to a quick rise in ICP to $100 \mathrm{~mm} \mathrm{Hg}$. At that moment, the flow stopped and reversal of flow into the tubing occurred. The experiment was repeated with similar results: when ICP rose to $30 \mathrm{~mm}$ $\mathrm{Hg}$, flow started to decline to a full stop over a 3-minute period, with a gradual rise in ICP to $38 \mathrm{~mm} \mathrm{Hg}$ (Fig. $3 \mathrm{~B})$. We found that as pressure increases, the BioMedicus pump flow gradually decreases to a stop and then causes flow reversal into the tubing, thereby providing a measure of safety that other irrigation systems do not provide. However, our results indicate that this built-in safety mechanism does not provide responses fast enough to prevent significant ICP elevations. Flow continued as long as ICP remained under $30 \mathrm{~mm} \mathrm{Hg}$. When ICP exceeded the critical value of $30 \mathrm{~mm} \mathrm{Hg}$, flow started to slow but with continued though momentary rises in ICP. The brain requires nearly instantaneous responses to high flow, considering that seconds of extreme ICP elevations may cause significant neurological damage. Therefore, the BioMedicus pump safety mechanism is not fast enough to protect against ICP elevations in the face of obstructed outflow, which means that sound clinical judgment must be exercised and ventricular outflow must be absolutely maintained during intraventricular neuroendoscopy.

\section{Gravity and Pump Flow Into an Open System}

Finally, intracranial flow from traditional irrigation systems is often affected by gravity from the height of the bag. To determine whether irrigation bag height affects the BioMedicus flow rate into an open system that simu- 

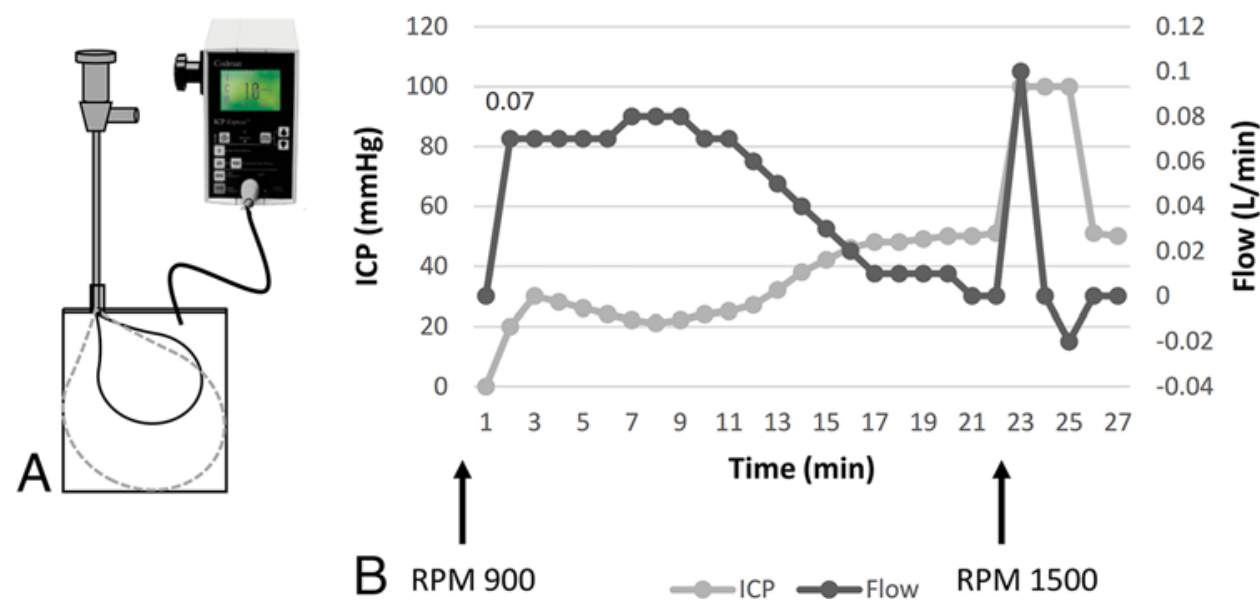

FIG. 3. Flow rate and canister pressure in a closed system. A: Closed system. An endoscope is connected to a closed 1200-ml canister that contains a balloon. The outflow track is blocked. A Codman ICP monitor is attached. The balloon is slowly inflated. The dotted line shows the balloon touching the inner walls of the canister at maximal expansion. B: Flow rate and ICP. In a closed system, as pressure increases, the BioMedicus pump flow decreases over a period of time to eventually stop and cause flow reversal into the tubing, providing a measure of safety that other irrigation systems do not provide. When ICP reached $30 \mathrm{~mm} \mathrm{Hg}$, the flow started to decline and eventually stopped after 8 minutes but with an accompanying gradual increase in ICP to $50 \mathrm{~mm} \mathrm{Hg}$. Subsequently, rpm was increased from 900 to 1500 to force fluid into the system at $100 \mathrm{ml} / \mathrm{min}$, which led to a quick rise in ICP to $100 \mathrm{~mm} \mathrm{Hg}$. At that moment, the flow stopped and reversal of flow into the tubing occurred. Figure is available in color online only.

lates clinical conditions, we placed the irrigation bag at different heights in relation to the canister (i.e., ventricle) level and induced flow at different rpm values while the ICP and flow rates were continuously recorded. When the bag was placed below or at the level of the canister, the flow rates measured were only those that the pump was programmed to deliver. When the irrigation bag was placed above the canister level with the pump turned off, flow was proportional to the bag height and attributed to gravity. When the pump was turned on, gravitational and pump flow were additive. Therefore, we found that gravity augments the flow rate delivered by the pump. Importantly, we learned that in all cases, as long as the bag height was not altered, the flow rate remains stable and ICP remains within a safe range. We concluded that to maintain full control of the flow rate delivered, the irrigation fluid bag is best positioned at the level of the ventricles.

\section{Clinical Testing}

Once the BioMedicus pump was determined to achieve an acceptable degree of safety, we initiated surgical trials and kept detailed records of the first 11 patients, including minute-by-minute recording of the pump settings, flow rates, and vital signs.

Of the 11 patients in the trial, 7 underwent ETV, 1 underwent cerebral aqueductoplasty, 1 underwent resection of a colloid cyst of the third ventricle, and 2 underwent tumor biopsy. The BioMedicus pump provided satisfactory irrigation in all cases. There was no instance of significant pneumocephalus, because the pump served to exclude all air by sequestering it in the impeller head. Visualization was excellent and, because of the Y-connector and 2-bag design, irrigation bags were replaced seamlessly with no interruption in irrigation. There were no neurological complications.

In 2 teenaged patients who underwent ETV with septal fenestration, vital sign changes were noted as a result of temporary outflow blockage, but both cases reversed when outflow was restored. In 1 patient, the heart rate momentarily dropped from 110 to 80 beats/min over 15 minutes, while in the other patient there was a gradual elevation of both the heart rate (from 60 to 90 beats $/ \mathrm{min}$ ) and systolic blood pressure (from 90 to $140 \mathrm{~mm} \mathrm{Hg}$ ) over a period of 40 minutes. Reestablishment of outflow led to the immediate normalization of vital signs, and surgery was resumed in both instances with no further complications.

An important factor that arose during clinical testing relates to the size and design of the endoscope and its ports. The MINOP endoscope system includes 3 trocar (sheath) sizes. The trocar used for complex endoscopy measures 6 $\mathrm{mm}$ in diameter and contains a $2.8-\mathrm{mm}$ optical channel to accommodate the endoscope, a 2.2-mm working channel, a $1.4-\mathrm{mm}$ irrigation channel, and a $1.4-\mathrm{mm}$ overflow channel. We found that the introduction of an instrument into the MINOP scope does not narrow the diameter of the irrigation port and in turn does not alter the flow rate delivered at any particular rpm.

Conversely, the Olympus infant cystoscope measures $2.7 \mathrm{~mm}$ in diameter and consists of a trocar with no separate irrigation or instrument port. In this cystoscope, the irrigation fluid travels in the trocar around the endoscope. If an instrument (e.g., biopsy forceps or Fogarty balloon) is introduced through the side arm, it also travels in the trocar next to the endoscope. We noted that the introduction of an instrument into the same compartment as the endoscope and the irrigation fluid would decrease the space occupied by the fluid and in turn decrease the flow rate delivered by the pump. Specifically, when the rpm is set to deliver a flow rate of $70 \mathrm{ml} / \mathrm{min}$, the introduction of a 2-Fr Fogarty balloon decreases the flow rate to $30 \mathrm{ml} / \mathrm{min}$. Therefore, when the BioMedicus pump or any other irrigation system is used, particular attention should be given 
to the type and size of the endoscope and the design of its instrument and irrigation ports.

Thus, we concluded that it is critical to monitor flow rate instead of rpm. The flow rate determines the volume and speed of the fluid entering the ventricles, whereas rpm is only an indication of how fast the rotor is spinning. We established the ideal flow rates by 1) determining the minimum flow needed to prevent a bleeding intraventricular vessel from forming a clot, and 2) using our experience treating many endoscopic cases to identify an arc-like stream that seems to indicate the lowest flow rate needed to prevent the accumulation of blood in the ventricle. Video 1 displays the different flow rates used with each endoscope: $70-100 \mathrm{ml} / \mathrm{min}$ using larger-diameter endoscopes (MINOP) and 30-40 $\mathrm{ml} / \mathrm{min}$ using smaller-diameter endoscopes (Olympus Infant Cystoscope).

VIDEO 1. Video clip showing the different flow rates used with the 2 types of scopes employed in our practice: the MINOP endoscope and the Olympus endoscope. Copyright Bermans J. Iskandar.

Published with permission. Click here to view.

Video 2 shows an example of clot prevention in the setting of a bleeding vessel.

VIDEO 2. Video clip showing intraventricular endoscopy complicated by bleeding from a choroidal vessel. Note that constant irrigation prevented a clot from forming in the ventricle before the vessel was coagulated successfully. Copyright Bermans J. Iskandar. Published with permission. Click here to view.

By the date of the submission of this report, we have used the BioMedicus pump for intraventricular irrigation in 310 cases spanning a 9-year period. These include ETV (116 cases), resection or biopsy of tumors and cysts (92), membrane or septal fenestration (42), posterior fossa approach (25), intraventricular hemorrhage evacuation (11), antibiotic irrigation for infection (5), and other procedures (19) that include endoscopic assistance during shunt surgery or external ventricular drain placement, intraventricular exploration, and removal of a retained ventricular catheter. We observed that the pump provides consistent irrigation, even during long operative procedures. In addition, we identified no major morbidity, no significant ICP elevations, and no significant intracranial air accumulation attributable to the irrigation system. Ambient air can occasionally access and accumulate in the ventricle via the peel-away sheath through which the endoscope is introduced. In the present series, this rarely occurred during active irrigation but seemed to instead occur when irrigation was temporarily halted.

Refer to the Appendix for a brief guide of the steps of our technique.

\section{Discussion}

The BioMedicus pump adapts well to neuroendoscopic applications and has distinct advantages over traditional irrigation systems: namely, it provides a consistent and easily adjustable flow rate that is displayed on a digital readout, has high- and low-flow alarms, and has the ability to prevent air from entering the cranial cavity. We presently use the pump in virtually all neuroendoscopic procedures with the exception of flexible shunt endoscopy. However, we irrigate only when needed, in order to avoid the tem- porary complications seen with prolonged irrigation using any system, such as intraoperative tachycardia, electrolyte disturbance, postoperative headaches, seizures, focal neurological deficits, or mental status changes. ${ }^{5}$ For example, in a typical ETV case, irrigation may be used briefly to clear debris from the tip of the scope after entry into the ventricle, and thereafter irrigation is only used if bleeding occurs or the ventricles are small and need constant irrigation to keep them dilated during surgery. Conversely, in a tumor resection case, continuous irrigation is often necessary to clear blood and debris.

The primary concern with the introduction of new technology or the novel use of operating equipment is safety. With this in mind, we set out to simulate conditions that may be encountered during intracranial endoscopy and to evaluate the performance of the pump under controlled conditions. There are several important points that came to light during these investigations.

First, equipment testing in a laboratory setting confirms that monitoring outflow is essential when using the system for neuroendoscopy and that the pump safety mechanism is not sufficient to prevent against instantaneous rises in ICP and, accordingly, does not replace sound surgical judgment.

Second, when endoscopes with no separate irrigation channels are used (e.g., the Olympus Infant Cystoscope), introduction of an instrument into the working channel results in a decrease in the flow rate. This can easily be corrected by increasing pump rpm. Most importantly, however, removal of the instrument from the endoscope can result in an instantaneous increase in flow, thereby requiring the rapid adjustment of pump rpm. Reassuringly, however, equipment testing also showed that significant increases in pump rpm do not lead to increased pressure if ventricular outflow is maintained. This provides a measure of assurance that the introduction and removal of instruments is unlikely to significantly affect ICP, even if flow changes dramatically. Notably, as far as we know, no other irrigation system in current use in neuroendoscopy provides intraoperative readouts of flow rate.

Centrifugal pumps have gained popularity in cardiac surgery mainly because, unlike other systems (e.g., roller pumps), they are not susceptible to overpressurization of the tubing if the tubing distal to the pump is kinked or clamped. ${ }^{3}$ If, instead, the tubing proximal to the centrifugal pump is kinked, large negative pressures can still be generated. In addition, because these pumps are nonocclusive, retrograde flow can result from the reduction in forward flow, such as when the impeller stops spinning or if the pump is too slow to generate the fluid pressure needed to maintain forward flow. ${ }^{3}$ In the setting of a cardiac procedure, such malfunctions can cause exsanguination of the patient ${ }^{3,6}$ However, in neuroendoscopic surgery, reduction in forward flow may cause the ventricle to collapse, which is a minor problem that can be prevented by clamping the line. In addition, because the flow rate can change in response to line obstruction, the use of a flow meter is imperative for safe surgery. ${ }^{1,3}$ Finally, centrifugal pumps prevent large amounts of air from entering the intracranial cavity because the air gets trapped in the impeller head, but if more than $32-52 \mathrm{ml}$ of air enters the circuit the 
pump will deprime and stop pumping. . $^{3,4}$ Importantly, the majority $(73.3 \%)$ of cardiac perfusion accidents are due to human error, and only a minority $(19.5 \%)$ are due to device malfunction. ${ }^{3,7}$ Therefore, attention to detail and strict adherence to proper pump setup and operation are essential provisions to minimizing such malfunctions.

\section{Conclusions}

We modified the BioMedicus 550 centrifugal pump to provide continuous irrigation for intraventricular endoscopy. Equipment testing in a laboratory setting established the safety of the system, with the important proviso that ventricular outflow must be scrupulously maintained. Clinical testing confirmed the pump's safety and effectiveness in neuroendoscopic surgery and discerned important caveats related to endoscopic channel design and size. The BioMedicus pump system provides a continuous intraoperative flow rate readout with high- and low-flow alarms and alleviates the problems of inconsistent flow rates, air in the irrigation lines, and delays in changing irrigation bags. When accompanied by sound surgical judgment, the BioMedicus centrifugal pump is a safe and efficient alternative to the more traditional inflatable pressure bag, peristaltic pump, or serial compression options while providing distinct advantages.

\section{Appendix}

\section{Practical Guide for the Use of the BioMedicus Pump System in Intraventricular Neuroendoscopy}

1. Install 2 warm fluid bags with a Y-connector. The second bag is used to prevent flow interruption as the other bag empties.

2. Set the high- and low-flow alarms.

3. Ensure the absence of air in the line.

4. Ensure appropriate fluid outflow from the ventricle through a peel-away sheath, ventricular catheter, or other strategy.

5. Prior to the start of surgery, determine the acceptable flow rate.

6. Prior to the start of surgery, determine how the insertion of various endoscopic instruments affects the flow rate. As surgery progresses, closely monitor the flow rate when the instruments are inserted and removed.

7. Once the pump is set up, irrigate sparingly, i.e., turn irrigation off when it is not needed to dilate the ventricles or evacuate blood and debris.

\section{References}

1. Berki T, Gurbuz A, Isik O, Akkaya H, Bayezid O, Yakut C: Cardiopulmonary bypass using centrifugal pump. Vasc Surg 26:123-134, 1992
2. Cappabianca P, Cinalli G, Gangemi M, Brunori A, Cavallo LM, de Divitiis E, et al: Application of neuroendoscopy to intraventricular lesions. Neurosurgery 62 (Suppl 2):575-598, 2008

3. Eiki T, Raskin S, Nose Y: Blood pumps, in Gravlee GP, Davis RF, Utley JR, et al (eds): Cardiopulmonary Bypass: Principles and Practice, ed 2. Philadelphia: Lippincott Williams \& Wilkins, 2000, pp 37-48

4. Hessel EA, Hill AG: Circuitry and cannulation, in Gravlee GP, Davis RF, Utley JR, et al (eds): Cardiopulmonary Bypass: Principles and Practice. Baltimore: Williams \& Wilkins, 1993, pp 55-92

5. Ganjoo P, Sethi S, Tandon MS, Singh D, Pandey BC: Perioperative complications of intraventricular neuroendoscopy: a 7-year experience. Turk Neurosurg 20:33-38, 2010

6. Kolff J, McClurken JB, Alpern JB: Beware centrifugal pumps: not a one-way street, but a potentially dangerous "siphon". Ann Thorac Surg 50:512, 1990

7. Kurusz M, Wheeldon DR: Risk containment during cardiopulmonary bypass. Semin Thorac Cardiovasc Surg 2:400409, 1990

8. Piek J, Oertel J, Gaab MR: Waterjet dissection in neurosurgical procedures: clinical results in 35 patients. J Neurosurg 96:690-696, 2002

9. Souweidane MM: Endoscopic surgery for intraventricular brain tumors in patients without hydrocephalus. Neurosurgery 57 (4 Suppl):312-318, 2005

\section{Disclosures}

The authors report no conflict of interest concerning the materials or methods used in this study or the findings specified in this paper.

\section{Author Contributions}

Conception and design: Iskandar, Henry. Acquisition of data: Rocque, Henry. Analysis and interpretation of data: Koueik, Rocque. Drafting the article: Koueik, Rocque, Iskandar. Critically revising the article: Bragg. Reviewed submitted version of manuscript: Iskandar, Koueik, Henry, Bragg. Approved the final version of the manuscript on behalf of all authors: Iskandar. Administrative/technical/material support: Henry, Paul. Study supervision: Iskandar.

\section{Supplemental Information \\ Videos}

Video 1. https://vimeo.com/235549903.

Video 2. https://vimeo.com/235550872.

\section{Correspondence}

Bermans J. Iskandar, Department of Neurological Surgery, University of Wisconsin, 600 Highland Ave., K4/832, Madison, WI 53717. email: iskandar@neurosurgery.wisc.edu. 